\title{
The relationship between the size of a muscle afferent volley and the cerebral potential it produces
}

\author{
SIMON GANDEVIA, DAVID BURKE, BRIAN MCKEON \\ From the Unit of Clinical Neurophysiology, Department of Neurology, The Prince Henry Hospital, and the \\ School of Medicine, University of New South Wales, Sydney, Australia
}

SUMMARY This study examined the relationship between the size of an afferent neural input produced by electrical stimulation of the posterior tibial nerve at the ankle and the size of the early components of the evoked cerebral potential. For five of six subjects the first peak of the afferent neural volley recorded in the popliteal fossa was uncontaminated by either motor efferents or cutaneous afferents. This was established by measuring the conduction times of motor fibres in the posterior tibial nerve and cutaneous fibres in the sural and posterior tibial nerves over the ankle to popliteal fossa segment. It is likely therefore that the first peak of the afferent volley contained predominantly, if not exclusively, activity in rapidly conducting afferents from the small muscles of the foot. The size of the two earliest components of the cerebral potential did not increase in direct proportion to the size of the afferent volley which produced it. The early components of the cerebral potential reached a maximum when the responsible muscle afferent volley was less than $50 \%$ of its maximum.

Recordings of short-latency cerebral potentials to peripheral nerve stimulation are used routinely to assess the integrity of central somatosensory pathways in man (see, for example, references 1-7). The size of the early components of these potentials depends, in part, on the intensity of stimulation of the peripheral nerve and thus, presumably, on the size of the afferent volley. An increase in stimulus intensity results in an increase in size of the cerebral potential in animals ${ }^{8-10}$ and in $\operatorname{man}^{71-14}$ but the potential reaches a plateau, saturation occurring at submaximal stimulus levels. This may indicate either that all the peripheral afferent fibres contributing to the cerebral potential have been recruited, or that there is a non-linear relationship between the input from the peripheral nerve and the potential it produces at cerebral levels. Peripheral nerve recordings suggest that the latter mechanism probably occurs, at least for the cutaneous input from digital nerves (see fig 5 of reference 5).

Recent studies on human subjects have shown that activity in muscle afferents, produced by muscle

Address for reprint requests: Dr S Gandevia, Unit of Clinical Neurophysiology, The Prince Henry Hospital, Little Bay 2036, Sydney, Australia.

Received 2 February 1982. Accepted 7 March 1982. stretch, tendon percussion or electrical stimulation of the posterior tibial nerve, can evoke a reproducible cerebral potential. ${ }^{15-17}$ This demonstration is consistent with the view that afferents from muscle contribute to proprioception ${ }^{18-20}$ (for review see ref 21). Such a projection is also a prerequisite for the postulated proprioceptive transcortical reflex. ${ }^{22-25}$ However, no data exist on the inputoutput relationship of the muscle afferent projection to the cortex in man. Electrical stimulation of the posterior tibial nerve at the ankle produces synchronous activation of muscle and cutaneous afferents. The cerebral potential evoked by this stimulus is derived predominantly, if not exclusively, from rapidly conducting muscle afferents. ${ }^{15} 16$ There may be little or no cutaneous contribution to the earliest components because of active inhibition of the cutaneous volley by the more rapidly conducting muscle afferents, ${ }^{16}$ though cutaneous afferents may contribute to later components of the cerebral potential. The present study was undertaken to determine the relationship between the size of the muscle afferent input to the central nervous system and the short-latency cortical potential it produces.

\section{Methods}

Experiments were performed on six healthy adult subjects, who lay prone on a bed and remained passive, but awake, 
throughout the experimental procedures. Informed consent was obtained. Constant voltage stimuli of $0.2 \mathrm{~ms}$ duration were delivered to the posterior tibial and sural nerves at equivalent sites on either side of the ankle using bipolar surface electrodes. The stimulus repetition rate was about $2 \mathrm{~Hz}$. Cerebral evoked potentials were recorded using stainless steel needle electrodes inserted subcutaneously at the vertex (active) and at the forehead (reference). The vertex site was chosen for the active electrode because previous studies have shown that at this site potentials from the lower limb are maximal. ${ }^{712627}$ A cranial reference was chosen to minimise contributions from extracranial and subcortical midline structures as may be seen with a truly indifferent electrode. After amplification (gain $100000-200000)$ and filtering $(1.6 \mathrm{~Hz}-1.6 \mathrm{kHz})$ the neural activity was averaged for a $100 \mathrm{~ms}$ period after the peripheral nerve stimulus using a fixed-programme averager with automatic artefact rejection. The sampling rate was $5 \mathrm{kHz}$. Usually 256 or 512 sweeps were averaged, although longer averaging runs (to 2048 sweeps) were sometimes necessary to define the onset of cerebral activity, particularly at low levels of peripheral nerve stimulation (see below). Duplicate averages were run routinely to ensure reproducibility of the onset and of the early peaks of each potential. Latency measurements were made with the aid of a cursor. The amplitudes measured were from the onset of detectable activity to the peak of the first positive wave (onset-P1) and from the peak of the first positive wave to the peak of the first negative wave (P1N1). Measurements were also made of the area of positivity of P1 using the baseline activity as reference zero. Unless otherwise specified the term cerebral potential is used throughout this paper to refer to the first positive wave, in particular, the onset-P1 and $\mathrm{P} 1-\mathrm{N} 1$ deflections. Peripheral nerve potentials were recorded in the popliteal fossa with bipolar electrodes (interelectrode distance 40 $\mathrm{mm}$ ). The recording electrodes were positioned at the site of lowest threshold for stimulation of the tibial nerve. Amplification and averaging techniques were similar to those used for the cerebral potental.

In each subject, before recording the cerebral evoked potential, the following conduction velocities were measured: (1) the motor conduction velocity to abductor hallucis for the popliteal fossa to ankle segment, (2) the conduction velocity of the posterior tibial mixed nerve action potential for the ankle to popliteal fossa segment, (3) the sural nerve conduction velocity for the ankle to popliteal fossa segment. The sural value provided a measurement of the conduction time of the fastest cutaneous fibres from ankle to popliteal fossa, and hence an indirect estimate of the latency of the cutaneous component of the mixed nerve potential. ${ }^{15} 16$ The validity of this assumption has been established previously ${ }^{15} 16$ and was confirmed in three of the present subjects by the demonstration that, in the ankle to popliteal fossa segment, the most rapidly conducting afferents in the sural nerve have the same conduction velocity as posterior tibial cutaneous afferents from the hallux. The peripheral nerve and cerebral potentials were recorded in response to different intensities of stimulation of the posterior tibial nerve at the ankle. The first run used a strong level of stimulation which produced the maximum size of the first peak of the peripheral nerve potential. This level was just below pain threshold. Weaker levels of stimuli were then presented in random order.

\section{Results}

\section{CONTRIBUTIONS TO THE MIXED NERVE} POTENTIAL

Table 1 shows the conduction velocities of the fastest motor fibres in the posterior tibial nerve, the fastest fibres in the mixed nerve action potential and the fastest cutaneous fibres in the sural nerve, all measured for the ankle-popliteal fossa segment. In each subject the conduction velocity was greater for the mixed nerve potential (mean $56.7 \mathrm{~m} / \mathrm{s}$, range $50.6-63.6 \mathrm{~m} / \mathrm{s}$ ) than for either motor fibres to abductor hallucis (mean $48.4 \mathrm{~m} / \mathrm{s}$, range $42 \cdot 1-53.6$ $\mathrm{m} / \mathrm{s}$ ) or cutaneous fibres of the sural nerve (mean $48.3 \mathrm{~m} / \mathrm{s}$, range $45 \cdot 2-53 \cdot 6$ ). The results from a typical subject are shown in fig 1 . In addition, in three subjects the cutaneous posterior tibial volley elicited by stimulation of the digital nerves of the hallux was found to have the same conduction velocity in the ankle to popliteal fossa segment as the sural volley. These results are consistent with previous findings which demonstrated that the fastest conducting group of fibres in the posterior tibial nerve are afferents not of cutaneous origin. ${ }^{1516}$

In all subjects the mixed nerve action potential contained two peaks, the second of which was smaller. It occurred at the same latency as the peak of the sural nerve action potential (fig 1 , see also fig 4 in ref 15). Presumably this second peak resulted from activity in cutaneous afferents, slowly conducting muscle afferents and antidromically conducting efferents. The onset of the cutaneous afferent contribution to the bipeaked mixed nerve action potential was estimated from the latency of onset of the sural sensory action potential. For five of the six subjects the latency of onset of the sural potential was longer than the latency to the peak of the initial component of the mixed nerve potential. In addition, in all subjects, the latency to peak of the initial component was shorter than the conduction time for the fastest motor fibres. In one subject (table 1, subject 4) the conduction velocity of the fastest afferents in the posterior tibial nerve $(50.6 \mathrm{~m} / \mathrm{s})$ was only

Table 1 Conduction velocities $(\mathrm{m} / \mathrm{s})$

\begin{tabular}{llll}
\hline Subject & Mixed CV & Motor CV & Sural CV \\
\hline 1 & $57 \cdot 2$ & $50 \cdot 0$ & $49 \cdot 1$ \\
2 & $57 \cdot 3$ & $48 \cdot 6$ & 46.6 \\
3 & 63.6 & $53 \cdot 6$ & $53 \cdot 6$ \\
4 & $50 \cdot 6$ & $42 \cdot 1$ & $47 \cdot 1$ \\
5 & $57 \cdot 5$ & $52 \cdot 0$ & $45 \cdot 2$ \\
6 & $53 \cdot 8$ & $44 \cdot 6$ & 47.9 \\
average & 56.7 & 48.4 & $48 \cdot 3$ \\
\hline
\end{tabular}




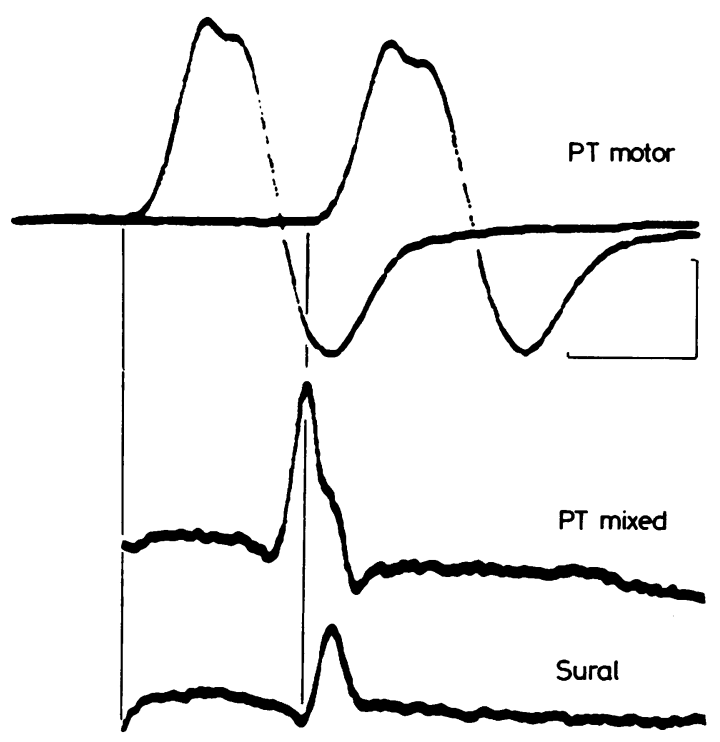

Fig 1 The upper trace shows the compound muscle action potential of abductor hallucis after stimulation of the posterior tibial nerve at the ankle and at the popliteal fossa. The middle trace shows the mixed nerve action potential recorded in the popliteal fossa after stimulating the posterior tibial nerve at the ankle. The lower trace shows the sensory action potential recorded in the popliteal fossa evoked by stimulating the sural nerve at the ankle. The lower two potentials have been aligned (left vertical line) with the onset of the electromyogram produced by a posterior tibial nerve stimulus at the ankle. A second vertical line (right) is drawn to show that the peak of the mixed nerve action potential has a conduction time which is shorter than that of either the cutaneous fibres in the sural nerve, or of motor fibres in the posterior tibial nerve over the ankle to popliteal fossa segment. These results are from subject 3 in tables 1 and 2. Calibrations: horizontal, $5 \mathrm{~ms}$; vertical, upper trace $7.5 \mathrm{mV}$, lower trace $3 \mathrm{uV}$. In this and subsequent figures a negative potential is shown as an upward deflection.

slightly greater than that of sural cutaneous fibres $(47 \cdot 1 \mathrm{~m} / \mathrm{s})$, such that a cutaneous contribution to the first peak of the mixed nerve action potential was likely. The cerebral evoked potentials recorded in this subject was not noticeably different from those recorded in the other subjects.

It is concluded that the initial peak of the mixed nerve action potential represents a pure afferent volley and that for five of the six subjects the peak was predominantly if not exclusively a muscle afferent volley. The size of the muscle afferent input was quantitated in two ways, by measurement of the area under the rising phase to the first peak of the mixed nerve potential and by measurement of the amplitude of the potential. Both methods gave similar results; only the latter measurements are illustrated in the figures.
CORTICAL EVOKED POTENTIALS

Latencies from individual subjects for the onset, P1 and N1 of the cerebral evoked potential are shown in table 2. For each subject the mean values in table 2 are based on 4-8 potentials. There was little alteration in latency throughout the experiment in an individual subject even with low intensities of stimulation. The prominent feature of the potentials evoked in all subjects was that the amplitude of their components did not increase in parallel with the size of the muscle afferent volley. At low stimulus levels the amplitude of the cerebral potential (onset-P1 and $\mathrm{P} 1-\mathrm{N} 1$ ) increased with increasing size of the input to the central nervous system when only a small proportion of muscle afferents were activated, but not at higher stimulus intensities when the majority of muscle afferents were activated (figs 2, 3). The size of these components reached a plateau when the muscle afferent volley was about half its maximal value. Results from all subjects are shown in fig 4 (at left: for onset-P1; at right: for P1-N1). Both early components of the cerebral evoked potential showed a comparable negative acceleration with increasing afferent input.

For the group of subjects, at a stimulus intensity which produced an afferent input of $10 \%$ of maximum, the $\mathrm{P} 1-\mathrm{N} 1$ component had reached an average $49.5 \%$ of its maximum value and at a stimulus intensity which produced an afferent input of $50 \%$ of maximum, the $\mathrm{P} 1-\mathrm{N} 1$ component had reached an average of $93 \%$ of its maximum. Comparable results were obtained when the afferent input and cerebral response were measured using areas rather than amplitudes.

In all subjects, low stimulus levels produced a nerve volley of low amplitude with only a single peak, occurring at the same latency as the first peak produced by strong stimuli (fig 2 , left). The cerebral potentials evoked by these low stimuli had the same latencies as those evoked by stronger stimuli (fig 2 , right; table 2), a finding which confirms that the cerebral potential resulted from activity in rapidly conducting muscle afferents. Indeed, in four of the present subjects it has been established that selective stimulation of muscle afferents using a microelectrode inserted into appropriate fascicles of the

Table 2 Latencies of cerebral potential (mean, SD in ms)

\begin{tabular}{llll}
\hline Subject & Onset & $P 1$ & $N 1$ \\
\hline 1 & $31.9(0.6)$ & $37.3(0.6)$ & $46.5(2 \cdot 1)$ \\
2 & $32.7(0.1)$ & $36.9(0.4)$ & $44.9(1.4)$ \\
3 & $31.7(0.6)$ & $37.6(1.0)$ & $44.3(1.3)$ \\
4 & $36.9(0.8)$ & $42.2(0.5)$ & $49.2(0.9)$ \\
5 & $32.1(0.4)$ & $37.5(0.8)$ & $44.2(0.6)$ \\
6 & $32.9(0.5)$ & $37.8(0.6)$ & $50.2(0.7)$ \\
average & 33.0 & 38.2 & 46.6 \\
\hline
\end{tabular}




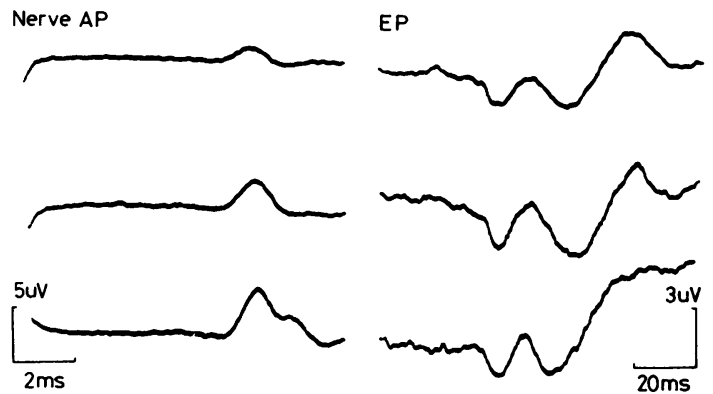

Fig 2 The results in subject 3 of three levels of stimulation of the posterior tibial nerve at the ankle. The mixed nerve action potential recorded in the popliteal fossa is on the left and the corresponding cerebral potential on the right. While there is a marked increase in the first peak of afferent activity there is only a modest increase in size of the early components of the cerebral potential and no change in their latencies. There is little alteration in the cerebral evoked potential when the level of stimulation was such that a second peak appeared in the mixed nerve potential.

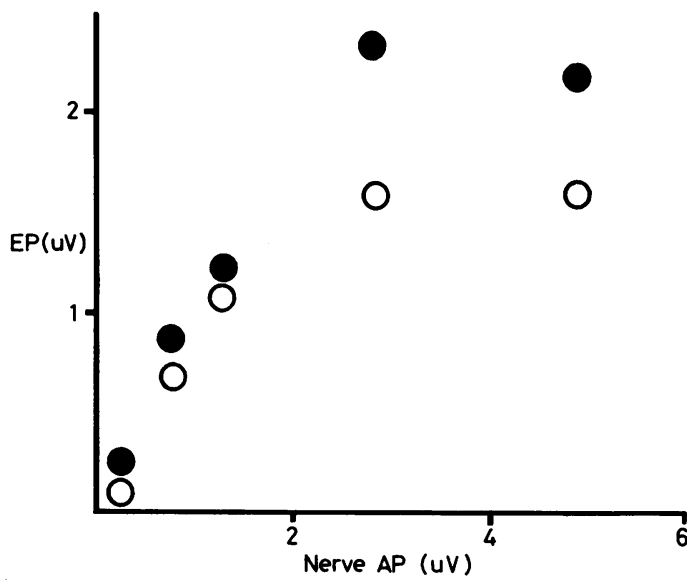

Fig 3 The amplitude of the early components of the cerebral potential evoked by stimulation of the posterior tibial nerve at the ankle is plotted against the size of the first peak of afferent activity in the mixed nerve potential in subject 3 (see text). Open circles represent the onset-P1 amplitude and filled circles represent the $P 1-N 1$ amplitude. The amplitudes of the cerebral potentials saturated for a submaximal neural input.

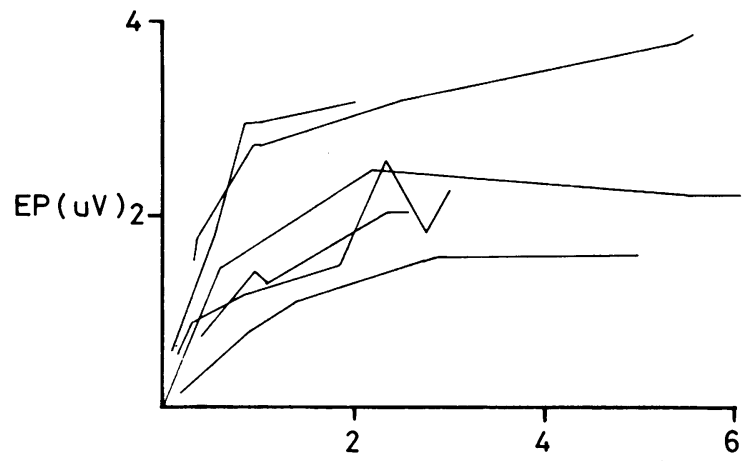

Nerve AP ( uV )

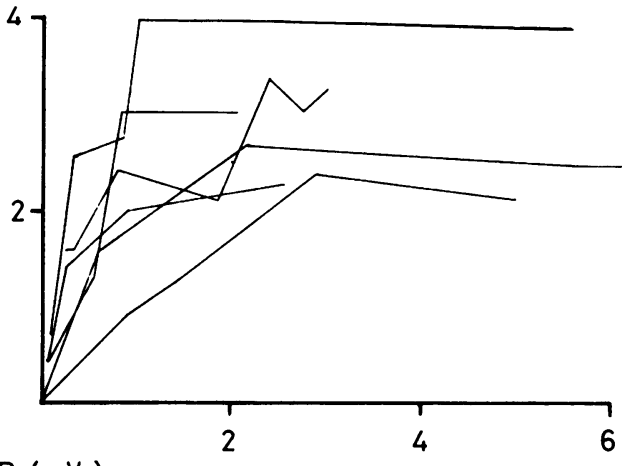

Fig 4 The results from six subjects are shown. Onset-P1 amplitudes of the cerebral potential are plotted at left and PI-N1 amplitudes are plotted at right, against the amplitude of the mixed nerve action potential evoked by stimulation of the posterior tibial nerve at the ankle.

posterior tibial nerve produces a cerebral potential of similar waveform and latency to that produced by surface stimulation. ${ }^{1516}$

It was noted consistently that, when the early components of the cerebral evoked potential were almost maximal $(>90 \%)$, further increases in stimulus intensity produced significant augmentation of the second peak of the peripheral nerve potential but little change in the early components of the cerebral potential. This is additional evidence that the early component of the cerebral potential evoked by stimulation of the mixed posterior tibial nerve does not contain a significant cutaneous component. ${ }^{1516}$

\section{Discussion}

This study describes a complex input-output relationship for the most rapidly conducting afferents in the posterior tibial nerve and the cerebral potential they produce. The afferents recruited at a low intensity of stimulation produce a short-latency potential which fails to increase proportionately as more afferents are recruited. Saturation of the early com- 
ponents of the cerebral potential can occur at intensities of stimulation which have activated as few as half the afferent fibres which produce the potential. This is so whether peak or areal measurements of the size of the afferent input are made. The conduction velocity of the afferent volley which produces the cerebral potential excedes that of motor axons and cutaneous afferents: the relevant afferent volley is therefore likely to be largely a group I muscle afferent volley. As the afferents from primary muscle spindle endings and from Golgi tendon organs have a similar diameter they would presumably be recruited in parallel with increasing intensity of stimulation. A recent study in man has shown that group Ia and group Ib afferents have similar conduction velocities and electrical thresholds. ${ }^{28}$ Thus the afferent input recorded in the popliteal fossa is almost certainly a heterogeneous one. However this afferent heterogeneity cannot be responsible for the observed saturation of the cerebral potential because electrical stimulation recruits group Ia and Ib afferents in parallel rather than in sequence.

Saturation of the early components of the cerebral evoked potential with increasing neural input has been described previously for cutaneous nerves in animals ${ }^{89}$ and man (see fig 5 of reference 12). It may also occur for the potentials evoked by stimulation of the human median nerve at the wrist. ${ }^{29}$ However, in this latter study ${ }^{29}$ no attempt was made to determine the relative afferent and efferent contributions to the mixed nerve potential or whether the afferent contribution was of predominantly cutaneous or muscle origin. The present results indicate that the muscle afferent projection also shares the phenomenon of "saturation" but they do not indicate whether the saturation involves the group Ia, Ib or both afferent projections. Whether or not this phenomenon has important perceptual or motor consequences is not revealed by the experiments reported here.

The neural mechanisms underlying the saturation phenomenon are obscure and have not been systematically studied. Possible factors include the following: first, the afferent fibres of largest diameter within a modality may traverse a pathway to cortex with greater convergence and synaptic efficiency. This would ensure relatively greater activation of higher order neurons. Second, within the somatosensory pathway, the volley from the most rapidly conducting afferents may inhibit or induce refractoriness to the input from less rapidly conducting afferents. That such a mechanism can occur is suggested by a recent study which demonstrated that the early components of cerebral potentials may be abolished by conditioning activity'in nerves from the same limb. ${ }^{16}$ Third, a more complex combination of convergence and "surround inhibition" may operate to channel the earliest activity along the somatosensory pathway. Whichever of these neural mechanisms operate, the somatosensory pathway seems capable of "amplifying" signals set up in only a few afferent fibres. It is interesting in this regard that cortical potentials can occasionally be recorded in response to stimuli too low to produce a detectable peripheral nerve potential in surface recordings, even after averaging many sweeps. As a consequence, cortical evoked potentials have been used to assess peripheral conduction in some hereditary neuropathies in which it is difficult to record peripheral nerve potentials. ${ }^{3}$

The results presented here have implications for diagnostic studies using evoked potentials. It should not be assumed that the early components of the cerebral potential evoked by stimulation of a mixed peripheral nerve have a cutaneous component or that the size of its early components necessarily reflects the size of the afferent volley which reaches the spinal cord. The use of intense stimuli in the belief that they are required to evoke the largest cerebral potential is unnecessary. Furthermore, stimulus strengths which produce maximal cerebral potentials are inappropriate for studying processing mechanisms in the somatosensory pathway. Both facilitatory and inhibitory interactions will be more easily detected if stimuli are such that they produce cerebral potentials which are clearly submaximal. In this respect, the apparent resistance of the early components of the cerebral evoked potential to modification by changes in stimulus rate, attention and anticipation could, in part, result from the use of relatively strong stimuli which produce a maximal cerebral potential that is difficult to modify.

With understanding of these principles and consideration of the relationship between the size of afferent input and the massed cerebral activity it produces, stimulation of the posterior tibial nerve may be useful for studying properties of the muscle afferent projection to cortex in man.

This study was supported by a grant from the National Health and Medical Research Council of Australia. The authors are grateful to Drs JW Lance and AK Lethlean for their support and comments on the manuscript.

\section{References}

${ }^{1}$ Cracco RQ, Cracco JB. Somatosensory evoked potentials in man: far-field potentials. Electroencephalogr Clin Neurophysiol 1976;41:460-6.

${ }^{2}$ Desmedt JE. Somatosensory cerebral evoked potentials in man. In: Rémond A, ed. Handbook of Electroence- 
phalography and Clinical Neurophysiology. Amsterdam: Elsevier 1971:vol 9, 55-82.

${ }^{3}$ Desmedt JE, Noël P. Average cerebral evoked potentials in the evaluation of lesions of the sensory nerves and of the central somatosensory pathway. In: Desmedt JE, ed. New Developments in Electromyography and Clinical Neurophysiology. Basel: Karger 1973:vol 2, 353-71.

${ }^{4}$ Desmedt JE, Cheron G. Central somatosensory conduction in man: neural generators and interpeak latencies of the far-field components recorded from neck and right or left scalp and earlobes. Electroencephalogr Clin Neurophysiol 1980;50:382-403.

${ }^{5}$ Giblin DR. Somatosensory evoked potentials in healthy subjects and in patients with lesions of the nervous system. Ann NY Acad Sci 1964;112:93-142.

- Starr A. Sensory evoked potentials in clinical disorders of the nervous system. Ann Rev Neurosci 1978;1: 103-27.

${ }^{7}$ Tsumoto T, Hirose N, Nonaka S, Takahashi M. Analysis of somatosensory evoked potentials to lateral popliteal nerve stimulation in man. Electroencephalogr Clin Neurophysiol 1972;33:379-88.

${ }^{8}$ Mark RF, Steiner J. Cortical projection of impulses in myelinated cutaneous afferent nerve fibres of the cat. J Physiol (Lond) 1958;142:544-62.

${ }^{9}$ Rosner BS, Schmid E, Novak S, Allison JT. Responses at cerebral somatosensory I and peripheral nerve evoked by graded electrocutaneous stimulation. $\mathrm{Am} \mathrm{J}$ Physiol 1959;196:1083-7.

${ }^{10}$ Schieppati M, Ducati A. Effects of stimulus intensity, cervical cord tractotomies and cerebellectomy on somatosensory evoked potentials from skin and muscle afferents of cat hind limb. Electroencephalogr Clin Neurophysiol 1981;51:363-72.

"Dawson GD. The relative excitability and conduction velocity of sensory and motor nerve fibres in man. $J$ Physiol (Lond) 1956;131:436-51.

${ }^{12}$ Debecker J, Desmedt JE. Les potentiels évoqués cérébraux et les potentiels de nerf sensible chez l'homme. Acta Neurol Belg 1964;64:1212-48.

${ }^{13}$ Small DG, Beauchamp M, Matthews WB. Subcortical somatosensory evoked potentials in normal man and in patients with central nervous system lesions. In: Desmedt JE, ed. Clinical Uses of Cerebral, Brainstem and Spinal Somatosensory Evoked Potentials. Prog Clin Neurophysiol. Basel: Karger 1980; vol 7, 190204.

${ }^{14}$ Rushton DN, Rothwell JC, Craggs MD. Gating of somatosensory evoked potentials during different kinds of movement in man. Brain 1981;104:465-92.

${ }_{15}$ Burke D, Skuse NF, Lethlean AK. Cutaneous and mus- cle afferent components of the cerebral potential evoked by electrical stimulation of human peripheral nerves. Electroencephalogr Clin Neurophysiol 1981;51:579-88.

${ }^{16}$ Burke D, Gandevia SC, McKeon B, Skuse NF. Interactions between cutaneous and muscle afferent projections to cerebral cortex in man. Electroencephalogr Clin Neurophysiol 1982;53:349-60.

${ }^{17}$ Starr A, McKeon B, Skuse NF, Burke D. Cerebral potentials evoked by muscle stretch in man. Brain 1981;104:149-66.

${ }^{18}$ Eklund G. Position sense and state of contraction: the effects of vibration. J Neurol Neurosurg Psychiatry 1972;35:606-11.

${ }^{19}$ Gandevia SC, McCloskey DI. Joint sense, muscle sense, and their combination as position sense, measured at the distal interphalangeal joint of the middle finger. $J$ Physiol (Lond) 1976;260:387-407.

${ }^{20}$ Goodwin GM, McCloskey DI, Matthews PBC. The contribution of muscle afferents to kinaesthesia shown by vibration-induced illusions of movement and by the effects of paralysing joint afferents. Brain 1972;95: 705-48.

${ }^{21}$ McCloskey DI. Kinesthetic sensibility. Physiol Rev 1978;58:763-820.

${ }^{22}$ Evarts EV. Motor cortex reflexes associated with learned movement. Science 1973;179:501-3.

${ }^{23}$ Lee RG, Tatton WG. Motor responses to sudden limb displacement in primates with specific CNS lesions and in human patients with motor system disorders. Can J Neurol Sci 1975;2:285-93.

${ }^{24}$ Marsden CD, Merton PA, Morton HB. Is the human stretch reflex cortical rather than spinal? Lancet 1973;i:759-61.

${ }^{25}$ Phillips CG. Motor apparatus of the baboon's hand Proc Roy Soc Ser B 1969;173:141-74.

${ }^{26}$ Eisen A, Elleker G. Sensory nerve stimulation and evoked cerebral potentials. Neurology (Minneap) 1980;30:1097-1105.

${ }^{27}$ Jones SJ, Small DG. Spinal and sub-cortical evoked potentials following stimulation of the posterior tibial nerve in man. Electroencephalogr Clin Neurophysiol 1978;44:299-306.

${ }_{28}^{28}$ Pierrot-Deseilligny E, Morin C, Bergego C, Tankov N. Pattern of group I fibre projections from ankle flexor and extensor muscles in man. Exp Brain Res $1981 ; 42: 337-50$

${ }^{29}$ Lesser RP, Koehle R, Lueders H. Effects of stimulus intensity on short latency somatosensory evoked potentials. Electroencephalogr Clin Neurophysiol 1979;47:377-82. 